(C) 2019 IEEE. Personal use of this material is permitted. Permission from IEEE must be obtained for all other uses, in any current or future media, including reprinting/republishing this material for advertising or promotional purposes, creating new collective works, for resale or redistribution to servers or lists, or reuse of any copyrighted component of this work in other works. 


\title{
Water Take-off and Landing Hybrid Copter approach for Maritime CONOPs
}

\author{
João Galante ${ }^{1}$, Manuel Ribeiro ${ }^{1}$, Roberto de Nobrega ${ }^{1}$, Jorge Neiva ${ }^{1}$, \\ António Sérgio Ferreira ${ }^{1}$ and João Borges Sousa ${ }^{1}$
}

\begin{abstract}
With the rise in the use of multi-vehicles teams, for maritime operations, new challenges and opportunities arise regarding the complexity and logistics of these scenarios. One way to cope with said complexity is to imbue some of these systems with the versatility of operating in more than one physical medium (air/water/land) during its normal mission cycle, maximizing their possible mission roles. The ability of having a vehicle which can operate both in the air and on the water can further expand and facilitate maritime operations by allowing new sampling, deployment and even communication scenarios. This work follows the iterations of a specific vehicle concept, through its various phases, and tracks the developments and challenges necessary to adapt a Remotely Piloted Aircraft Systems (RPAS) to become capable of water take-off \& landing, and explores its applicability as a viable operational mobile communication gateway for underwater and surface assets.
\end{abstract}

\section{INTRODUCTION}

Even today, the complexity in undertaking maritime operations is still an issue that has no clear and defined playbook. A vast majority of operations are still carried out by manned ships, making them the de facto workhorse of seagoing operations. Nevertheless, a ship can only be at one place at a time, can only carry a limited number of working personnel, and can only stay at sea for a limited amount of time. With our increasing need to understand and monitor how key maritime issues [1] steps must be taken to streamline and facilitate our ability to operate in this environment. One way to address this issue has been through the ongoing use of autonomous systems, both aerial, surface and underwater [2], [3], [4], [5], working in tandem with each other as a team. The use of teams of autonomous systems has not only allowed for easier operational logistics, but also expanded what can be done within the maritime environment [6], [7], [8]. Nevertheless, deployment these teams remain a challenging endeavor [9], [10] which has, on the most part, been mitigated by either the incorporation of mixedinitiative behavior [11], [12], [13] or by taking extensive care in regards to the way human operators interact with them [14], [15].

However, an important factor for the effective usability of these teams of systems, which isn't directly covered by expanding their levels of autonomy or improving their interfaces, is the capability of these teams exchanging and

\footnotetext{
${ }^{1}$ LSTS - Laboratório de Sistemas e Tecnologia Subaquática, Dept. of Electrical and Computer Engineering, School of Engineering (FEUP), University of Porto, Portugal $\{$ galante, maribeiro, rnobrega, jneiva, asbf $\}$ @lsts.pt; jtasso@fe.up.pt
}

relaying data between each other and the outside [4], [16], [17]. Issues of communication between these heterogeneous systems are paramount not only for coordination, especially when working in tandem with manned system [18], [19], but also for in-situ data collection and analysis. These issues further compound when taking into account that in maritime operations there is a mixture of communication channels, protocols and frequencies which must coexist in a collaborative fashion, to maximize data flow.

One way to facilitate these types of operations can be achieved by the inclusion of a system with the capacity of performing activities in more than one medium (air and water). This system, if able to operate as a hybrid element in the autonomous vehicle team, can fill-in gaps as needed while, at the same time, have its own specific tasks besides operation support. In this regard, a copter type RPAS presents itself as a promising starting system to adapt for aquatic operations, while minimizing side-effects to its effectiveness as an aerial vehicle. Its capacity to control its ascent and decent with precision is a benefit for any water approaches while, at the same time, its typically regularshaped horizontal cross-section allows for easy mounting and customization for different payloads.

The paper is structured as follows. In section II we present the challenge this paper addresses, as well as the scenarios in which it presents itself. Consequently in Section III we showcase the background work done at LSTS which allows for the current baseline of developments and testing. Section IV describes the conceptual idea behind the RPAS system, beginning by documenting the necessary changes to the RPAS frame and the payload system. It further expands by detailing implementation choices and the challenges behind making a working prototype. In section VI we present some field experiments along with lessons learned. Finally, in section VII we talk about future developments and some conclusions are drawn.

\section{MOTIVATION}

The motivation for the present work originated from an increasing need, in multi-vehicle operations, to reduce and manage the logistical footprint. As the amount of vehicles and specific payloads, deployed simultaneously, increase so does the operation cost and complexity. The ability of having a system which can perform multiple roles in separate physical mediums allows for a reduction in this complexity, as long as the command and control software allows for dynamic re-tasking of the asset. However, the vehicle itself 
must be designed/adapted in such a way that makes it compliant with multiple payload sorties, and so, modularity is a key factor during developments.

A starting point for development had to be chosen and, taking into account the operational context at the Laboratório de Sistemas e Tecnologia Subaquática (LSTS), focus was given to networking and communication improvements in the maritime scenario theatre. Priority was given to bestow the RPAS with the capability of functioning as a mobile communication gateway with the ability of multiplexing between different physical channels simultaneously. With this capability, re-tasking and re-planning can occur in more covert scenarios with little to no expose of underwater assets at the surface. Additionally, it allows for the collection and relaying of data from moored assets without the need for physical recovery of said assets.

Nevertheless, the biggest motivation remains the preparation of a platform which can employ multiple payloads, in a modular fashion, in real environments incorporated with autonomous vehicle networked teams.

\section{Contextualization}

All work was conducted at the LSTS, from University of Porto, which is specialized on the design, construction, and operation of unmanned underwater, surface and air vehicles and on the development of tools and technologies for the deployment of networked vehicle systems. The institution has, over the last 20 years, successfully fielded unmanned air, ground, surface and underwater vehicles in the Atlantic and Pacific oceans, and also in the Mediterranean and North seas. Moreover, its current active cooperation protocol with the Portuguese Navy allows for the deployment of large scale yearly exercises [20], [21], [22], [23] which serve as an operational testing event with active stakeholders and practitioners with the capability of validating the proposed concepts of operation (CONOPs).

\section{CONCEPT DESIGN}

By delving into what solutions were already being explored by other research groups, we found that a large majority were too conceptual, costly and of complicated implementation for our needs. A search began for off-theshelf solutions where we found a few options we identified as being an adequate starting point to make modifications and start developing our own system.

\section{A. Vehicle Base Frame}

Our previous attempt to build a RPAS capable of water landing [24] showed there several limitations of the chosen hexacopter platform, starting with the frame's small size and the difficulty in customization/adaptation to other payloads. Moreover, the thrust power available for that frame was very underwhelming and further limited its viability for more complex operations.

With that in mind, we went looking for a frame that could fulfill our needs, namely the possibility of having a larger payload capacity and acceptable flight times. The model we

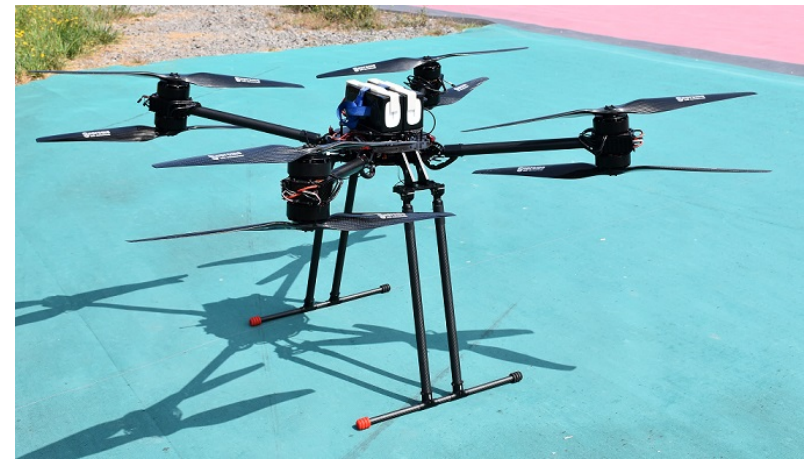

Fig. 1: D130 X8 Frame

ended up choosing was the D130 X8, sold by Foxtechfpv, octa-quad, with foldable arms, retractable landing gear and its 8 motors that provide much more power than the previous setup, giving not only more lifting capacity but also increased redundancy and stability (Fig. 1). Contrary to our previous build, which had limited usable frame space, this new iteration gives us more room for customization, over the space limitations we faced before.

\section{B. Flotation}

One major component in this project was the flotation device. Upon choosing the base platform, as described in IV-A, we made calculations for the estimated weight of the platform. This estimation placed the vehicle at approximately $20 \mathrm{Kg}$, meaning that, for freshwater, 20 liters of water displacement were needed to keep the frame afloat. Assuming the frame is ultimately targeted to work on seawater the same flotation device would still be capable of sustaining the vehicle. Furthermore, the calculations also accounted for the shift in the height of the center of mass for this scenario.

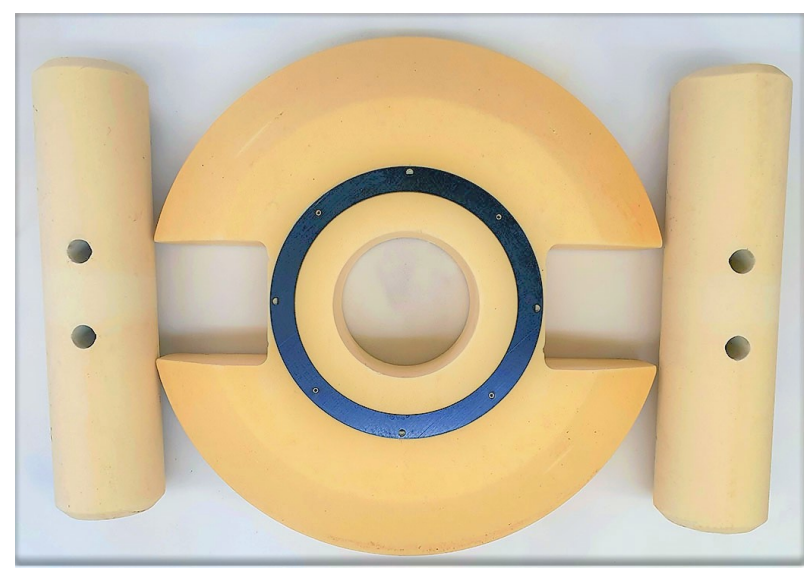

Fig. 2: Flotation Modules

After knowing the magnitude for the impulsion needed, the next problem was maintaining stability in the water and the clearance needed for the propellers. To make sure the vehicle could compensate for waves and recover during egress, the design should follow that of a platform-type flotation. Taking advantage of the retractable landing gear, present in the base 
frame, the flotation device was divided in three sub-parts (2), one main flotation at the center, where most of the impulsion is applied and two secondary flotation modules, at the end of each leg, grating extra stability to the platform, making sure it could recover from accentuated oscillations. A representation can be seen in 3 .

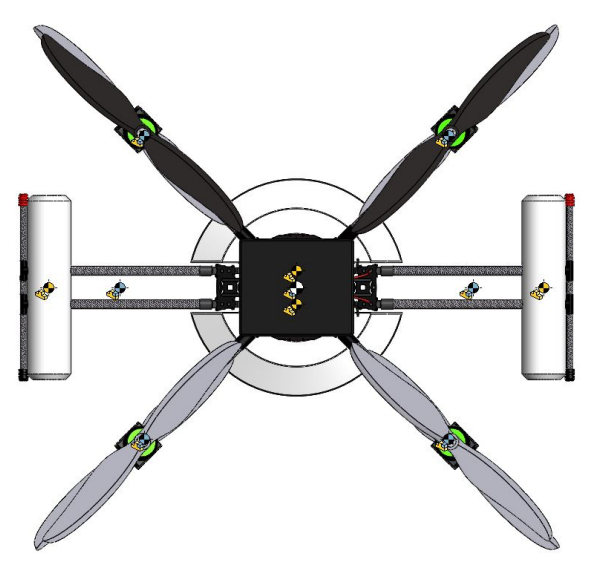

Fig. 3: Mass distribution for the platform in ingress/egress mode

One aspect that is crucial for this type of hybrid vehicle is maintaining a healthy trade-off between the copter performance in the air and in the water. A platform, to be stable in the water, needs the biggest area of contact possible and the lowest height for its center off mass. Ideally the center of mass would be lower then the center of impulsion, but for the copter's flight performance, the center of mass needs to be as close as possible to the point where the resulting force of the eight motors is applied, making the reaction to dynamic solicitation as good as the ESCs' response time and the power still available at that time.

Moreover, a copter wants the least frontal area possible, to reduce drag and limit the effects of wind during flight. Further complication for the development process, the height of the center of mass affects the clearance of the propellers when floating. Increasing it too much leads to a lack of stability during take off, to little and the propellers will hit the water. Both problems could end in catastrophic failure of the platform.

These conflicting requirements were one of the major platform adaptation challenges and after careful consideration and simulations we made the prototype presented in this paper. The flotation used was custom built to our specifications, after a series of design iterations, using Expanded Polyurethane Foam as base material, adding parts made of Polyacetal for structure and fastening purposes.

\section{Energy System}

In order to allow this platform to be serviceable in a maritime environment, its energy source and main electronic components needed to be sealed and protected at all times. Creating a sealing solution from scratch would be a tremendous endeavour therefore an off-the-shelf case (UltraBlox 312) was chosen to protect the batteries and power electronics used.

The batteries selected were two 6-cell 22Ah Lithium polymer (LiPo), that are connected in series. This configuration provides about $1108 \mathrm{~W}$ of power. To safely manage the power of the batteries, a new circuit board was created with the added premise of facilitating connection between the batteries and motors. Before the development of this board, market research was done and no satisfactory solution was found due to the maximum current the motors could draw.

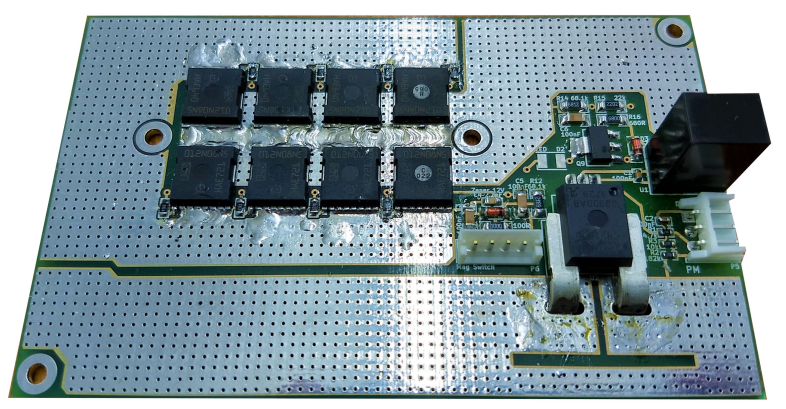

Fig. 4: Copter Safety Power Switch Board

That being said, we had to design our own solution and came up with the Copter Safety Power System board (CSPS) (Fig. 4). The solution is composed by a four layer Printed Circuit Board (PCB) using the IPT012N08N5 Metal Oxide Semiconductor Field Effect Transistor (MOSFET) from Infineon. These MOSFETs allow continuous currents of $300 \mathrm{~A}$, at room temperature, and a peak current of $1200 \mathrm{~A}$ [25].

Since this board is installed inside the same case as the batteries, the rise in temperature from the circuit could affect the health of the batteries. For this reason each motor has a dedicated MOSFET, maintaining temperature influence at its minimum to preserve battery life and efficiency. Finally the CSPS has a current measuring sensor which can read the motors' consumption and the battery voltage, allowing the flight controller to track these values during its mission.

Finally, in order to secure a connection between the power source and the ESCs, a dedicated connector was developed for the system's energy module, allowing conventional XT90 connectors to pass it while closing off water, making the assembly watertight, IP65 rated.

\section{Computational Stack and Pod}

The main components of the computational stack (Fig. 6) are as follows:

- CPU: A Raspberry Pi 3 running our onboard software, DUNE. This CPU is connected via serial port to the flight controller which enables it to exchange telemetry and commands with the flight controller.

- Flight Controller: A Pixhawk 2.1 running open source autopilot, in charge of attitude and position control of the platform with its internal redundant IMUs. 
- Wireless Link: An Ubiquiti Pico station $2.4 \mathrm{GHz}$ Wi$\mathrm{Fi}$ adapter for long range wireless communications. For pilot control, a FrSky X8R $2.4 \mathrm{GHz}$ radio module was also used, which is connected directly to the flight controller, giving pilot's override control.

- Ethernet Switch: A network switch that connects the CPU, the Wi-Fi link and any possible payloads.

- Payload System: A Seatrac X150 acoustic modem with USBL correction for underwater communications, which in perfect conditions has an acoustic range of $1 \mathrm{~km}$ radius both horizontally and vertically.

- Landing Gear Controller: Two separate controllers that allow the landing gear to extend and retract by means of a pilot-operated switch.

- Copter Power Management Board (CPMB) - our inhouse developed power circuit board, presented in IVC. Three DC/DC converters from manufacturer Traco Power were used on this PCB to generate $24 \mathrm{~V} @ 2 \mathrm{~A}$, 12V@2.5A and 5V@4A. These DC/DC converters were chosen because of their straightforward and simple integration with the designed system. With the CPMB the following payloads can be all connected at once:

- 5V, Pixhawk and Raspberry Pi

- 12V, Ubiquiti Pico-Station and Ethernet Switch

- 24V, Landing Gear and Acoustic Modem

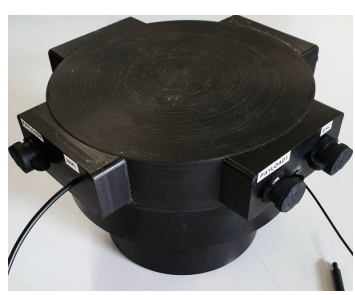

(a) Electronics Pod

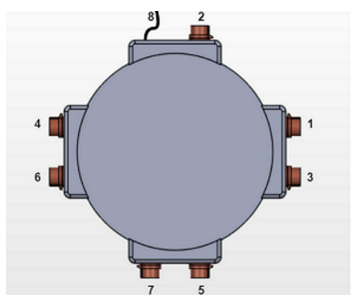

(b) Connections
Fig. 5: From concept to machined part

\begin{tabular}{|c|l|}
\hline Conn. \#: & Description: \\
\hline 1 & Main Power \\
\hline 2 & Payload 2 \\
\hline 3 & Left side ESCs \\
\hline 4 & Payload 1 \\
\hline 5 & GPS \\
\hline 6 & Right side ESCs \\
\hline 7 & Landing gear \\
\hline 8 & Wi-Fi antenna cable \\
\hline
\end{tabular}

TABLE I: Description of connections

With regard to the electronics stack connection options (Fig. 5 and Table I), the most relevant connectors are the Main Power input, that will provide power coming from the Energy System and the Payload 1 and Payload 2 connectors, that enable specific payloads to be plugged. With modularity in mind, both Payload 1 and 2 can be used in different configurations. As an example, Payload 1 can be used either to connect an acoustic modem or a telemetry radio while Payload 2 can be used to connect any miscellaneous payload, to the internal network switch.

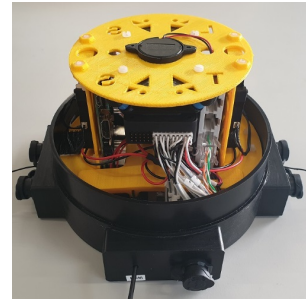

(a) Pod - Inside view

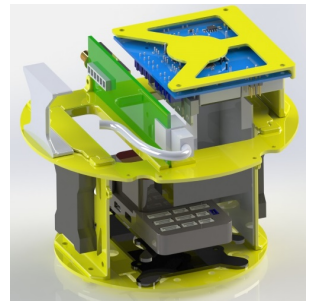

(b) Computational Stack CAD
Fig. 6: Computational Stack and Pod

\section{E. Acoustic Modem}

At this stage, the payload being used was an acoustic modem. The chosen model for this prototype was the SeaTrac $\mathrm{X} 150$. When compared to the previous approach [24], the Seatrac X150, adds features such as, USBL for a more robust solution for practical use.

The height of the modem, as seen in picture 7, was determined by the pressure at which the modem could be safely turned on. This pressure, translated to depth, was determined by the depth at which the modem can sense the variation in pressure, allowing for it to know when it is submerged and only then start transmitting.

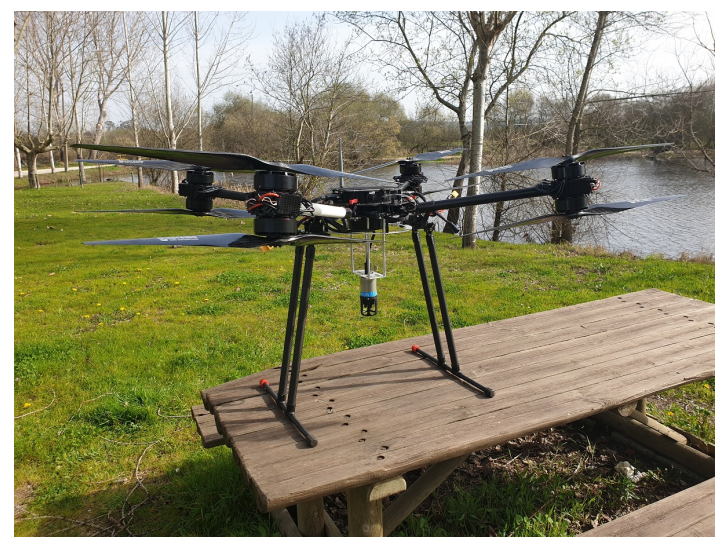

Fig. 7: Modem Payload installed

\section{F. Software}

For the low-level control of the platform, we rely on the open-source ArduCopter [26] latest software. It consists of navigation software running on the vehicle that offers fully autonomous, semi-autonomous and fully manual flight modes, programmable missions with $3 \mathrm{D}$ waypoints and integrates a large number of navigation sensors, including but not limited to GPSs, RTK GPSs, barometers, magnetometers, rangefinders, ADS-B transponder and airspeed sensors, and also implements useful fail-safe functions that trigger specific user-defined behaviour in cases of loss of pilot radio link, GPS failure, breaching a predefined operational area boundary or a minimum battery power level.

Nevertheless, in order to allow for the intended CONOPs the vehicle had to be incorporated into the LSTS software 
toolchain, detailed in [21]. Briefly, the toolchain itself is composed of 3 fundamental elements ranging from lowlevel control and navigation, up to the command and control interface. These elements are:

a) Dune: DUNE is the on-board software running on the vehicle, which is responsible not only for every interaction with sensors, payload and actuators, but also for communications, navigation, control, maneuvering, plan execution and vehicle supervision.

b) IMC: Inter-Module Communication (IMC) protocol [27] is a message-oriented protocol designed and implemented to build interconnected systems of vehicles, sensors and human operators that are able to pursue common goals cooperatively by exchanging real-time information about the environment and updated objectives.

c) Neptus: Neptus is the command and control software that allows operators to interact with networks of heterogeneous autonomous vehicles and sensors [28], [29], providing support for the entire mission-life-cycle: planning, simulation, execution and mission analysis. It can be adapted by operators to fit mission-specific requirements by adding vehicle-specific and mission-specific plug-ins to a console.

By connecting the flight controller to the CPU, by means of a wired telemetry link, one can control the platform using our own command and control interface Neptus, ranging from issuing new plans to live monitoring and interaction with other LSTS operational assets on the same network.

\section{Assembly Sequence \& Considerations}

The Titan platform is divided into a set of modules. Firstly there is the copter frame, as described in section IV. This module is the skeleton on which all other modules are built upon, it includes the landing gear, propellers, electronic speed controllers (ESC), motors and the overall structure of the vehicle. Secondly there is the Computational Stack module which is composed by the housing for the electronics, designed in-house, and the electronics it houses. Thirdly, is the Energy module, which is composed by Li-Po battery solution and a CSPS. The fourth module consists of the floating system and lastly is the payload module, in this example housing the acoustic Seatrack X150.

The computational stack is positioned in the middle of the base frame and between the power module and the payload, meaning that assembly wise it is imperative that the stack is the first module to be secured onto the base frame. The stack does posses an assembling orientation, having connectors number 5 and number 7 facing the front of the vehicle. Once the computational stack is firmly attached to the base frame, with the correct orientation, the power module can be assembled. This module lies on top of the base frame, above the computational stack. The opening of the power box should be oriented to the front of the vehicle, so that all connections are correctly aligned.

Following this, the arms of the copter can be elevated and screwed into place. Having the arms raised grants easy access to the center of the lower part of the vehicle to allow for the assembly of the flotation support ring and

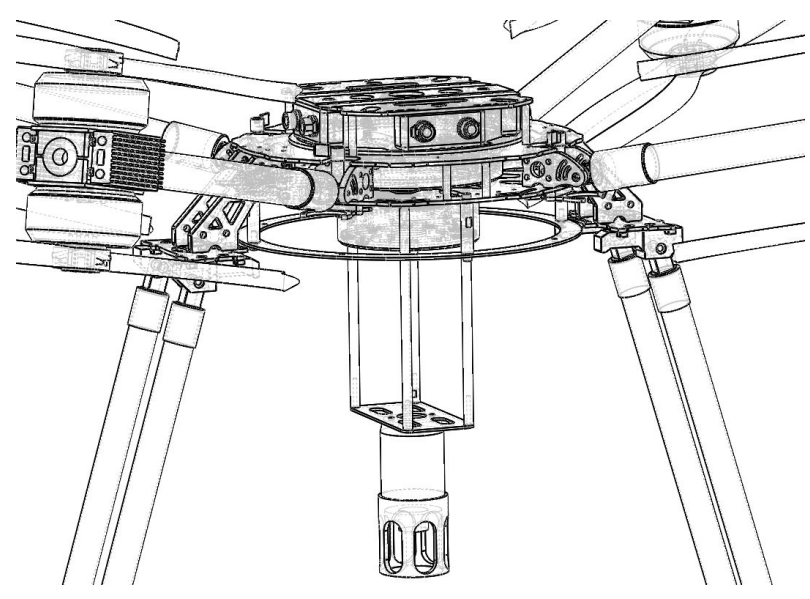

Fig. 8: Assembly Simulation

acoustic modem modules. It is important that the flotation support ring is assembled first and, thereafter, the acoustic modem module can be attached. The flotation support ring and acoustic modem module are placed in the center of the copter, precisely below the computational stack. These components should be secured before the propellers to avoid any physical damage to the users or the vehicle while accessing the lower segment of the frame. Only at this point, should all connections can be finalized, and connectors that are not being used should be sealed.

Subsequently, the eight propellers can be placed. The propellers are placed before securing the flotation foam modules, to the support ring and arms, to avoid any damage materials while securing the propellers to the motors.

Finally the flotation is assembled into place. The flotation module is composed of 3 pieces, as detailed before, with the center piece being screwed onto the support ring that was previously attached to the lower half of the vehicle. The central foam piece should be secured first given that it is the largest of the three pieces, requiring more space to handle. To assemble the lateral flotation pieces, one leg of the landing gear should be removed to be able to slide the flotation piece along the leg until it reaches the bottom. This leg can now be reassembled onto the frame, so that the same can be done for the remaining leg.

\section{FIELD EXPERIMENTS \& LESSONS LEARNED}

During the development of the platform tests were done in an iterative fashion, to all the different modules, within the molds of test-driven development (TDD). Initial testing was done within the lab but rapidly evolved to the field. This was possible in part due to our previous experience in this CONOP. However added care was taken when studying and testing the flotation module. In this regard flotation and stability in flight \& in water was done though the implementation of a Buckingham PI Theorem model to test geometry and materials. This model was used in a small pool, filmed and studied to help improve the design for the flotation. 
After these preliminary bench tests, the fist prototype was taken to a field location, for real life testing.

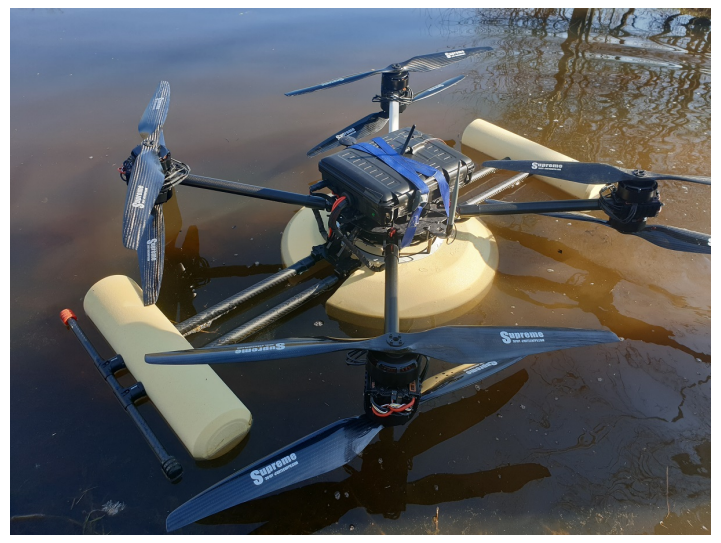

Fig. 9: Initial water test

The first day of field testing started with strenuous ground tests. After some adjustments to the height of flotation and testing the motors individually, the platform was ready for a basic flight test, measuring flight time and visually assessing the effects of carrying the flotation during regular copter flight. Furthermore, the vehicle had its landing gear actuated to ingress mode and, with the vehicle turned off, manually placed in the water. Following this procedure, a series of tests were preformed manually, in order to simulate the platform's response to the environment. The results confirmed the assumptions made during the development phase and gave reassurance to expand the testing complexity.

Nonetheless, some time was dedicated towards testing the separate modules of the platform and minor issues were found and promptly resolved. Moreover, the first ingress was attempted and recorded, ending in a successful water landing and validating the work described so far. Some damage was found in the secondary flotation devices, which was a result of a bent in the structure during transport. Better accommodation will be made for future transport to protect the prototype.

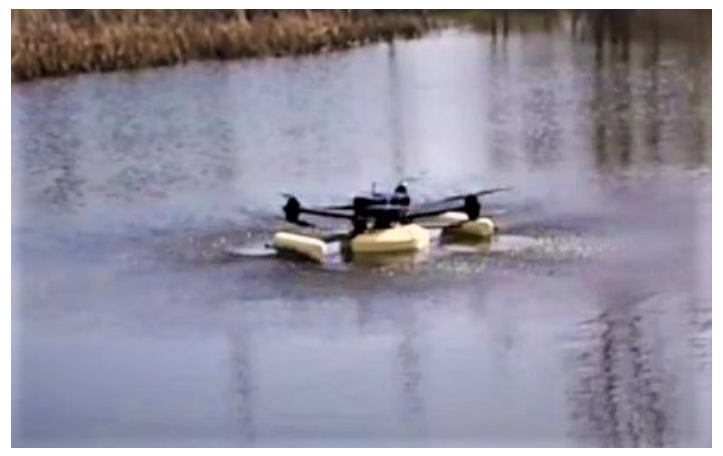

Fig. 10: First Water Take-off

Moving forward with the tests, a second attempt at water landing was performed, this time without any damage. The vehicle was then placed manually in the water, in order to minimize variables, and water take-off was a performed.
The experiment was successful and the vehicle behaved has expected during conception and development.

During the process of performing these tests, some problems and minor flaws were quickly identified and resolved. Per design, the water landing maneuver depends on the landing gear opening to a horizontal position. A defect in the distance between feet caused problems opening and closing the servos that control the landing gear, making it stuck in random positions. By failing to achieve one of the two possible landing positions it became impossible to land either in water or in land. A emergency landing was performed with same damage to the frame.

After repairs and a second trial at the take off maneuver, a full take-off from land, water ingress\&egress was performed, in order to test the full cycle.

Accordingly, the full maneuver of ingress and egress was performed over 14 individual flights with $100 \%$ success rate, fully corresponding with the expectations for this work.

\section{CONCLUSIONS \& FUTURE WORK}

Following on previous work, this paper presents the latest developments made with regards to a water-landing and takeoff capable platform. The Titan octocopter performed to expectations over the course of several individual flights. Additionally, the multiple modules implemented onto it added increasing functionality and reliability proving to be a natural upgrade to previous iterations of this project. The developed flotation solution delivered as expected, granting the necessary stability while floating, without affecting flight in a very pronounced way. Also, the vehicle was capable of carrying and using the acoustic modem payload, as intended. Additionally, this work documents the different steps and challenges that were encountered during the development and testing of this autonomous hybrid vehicle, ranging from component choices to assembly procedures and care.

Meanwhile, new payload systems are starting to be developed, notably, a water collecting system and a carry\&drop system and a generic Gimbal for specialized cameras payload is also in the works for this platform. Finally, this octocopter is scheduled to be tested this year in more complex scenarios of operation, as part of an integrated experiment between the LSTS, the Applied Research Laboratory at the University of Hawaii and elements of the US Naval Undersea Warfare Center (NUWC) with the end-goal of incorporating the Titan copter into a team of heterogeneous vehicles, running the LSTS toolchain while serving as a mobile relay station.

\section{ACKNOWLEDGMENTS}

This work was partially funded by the European Union's Horizon 2020 research and innovation programme under grant agreement No 731103 and MARINFO - Integrated Platform for Marine Data Acquisition and Analysis, supported by Norte Portugal Regional Operational Programme (NORTE 2020), under the PORTUGAL 2020 Partnership Agreement, through the European Regional Development Fund (ERDF). 


\section{REFERENCES}

[1] Ioc/Unesco, Imo, Fao, and Undp, "A Blueprint for Ocean and Coastal Sustainability," United Nations Conference on Sustainable Development, p. 42, 2011.

[2] L. L. Sousa, F. López-Castejón, J. Gilabert, P. Relvas, A. Couto, N. Queiroz, R. Caldas, P. S. Dias, H. Dias, M. Faria, F. Ferreira, A. S. Ferreira, J. Fortuna, R. J. Gomes, B. Loureiro, R. Martins, L. Madureira, J. Neiva, M. Oliveira, J. Pereira, J. Pinto, F. Py, H. Queirós, D. Silva, P. B. Sujit, A. Zolich, T. A. Johansen, J. B. de Sousa, and K. Rajan, "Integrated Monitoring of Mola mola Behaviour in Space and Time." PloS one, vol. 11, no. 8, p. e0160404, aug 2016.

[3] J. Fortuna, F. Ferreira, R. Gomes, A. S. Ferreira, and J. B. Sousa, "Using Low Cost Open Source UAVs for Marine Wild Life Monitoring - Field Report," in 2nd IFAC Workshop on Research, Education and Development of Unmanned Aerial Systems (2013), L. Rogelio, Ed. International Federation of Automatic Control, nov 2013, pp. 291295.

[4] A. G. Guerra, A. S. Ferreira, M. Costa, D. Nodar-López, and F. A Agelet, "Integrating small satellite communication in an autonomous vehicle network: A case for oceanography," Acta Astronautica, jan 2018.

[5] K. R. Skøien, M. O. Alver, A. P. Zolich, and J. A. Alfredsen, "Feed spreaders in sea cage aquaculture Motion characterization and measurement of spatial pellet distribution using an unmanned aerial vehicle," Computers and Electronics in Agriculture, vol. 129, pp. 2736, nov 2016.

[6] A. S. Ferreira, M. Costa, F. Py, J. Pinto, M. A. Silva, A. Nimmo-Smith, T. A. Johansen, J. B. de Sousa, and K. Rajan, "Advancing multi-vehicle deployments in oceanographic field experiments," Autonomous Robots, pp. 1-20, oct 2018. [Online]. Available: http:https://doi.org/10.1007/s10514-018-9810-x http://link.springer.com/10.1007/s10514-018-9810-x

[7] A. S. Ferreira, J. Pereira, P. Sousa Dias, J. B. de Sousa, T. A. Johansen, P. Lourenco, and V. E. Hovstein, "Managing communication challenges in vehicle networks for remote maritime operations," in OCEANS 2017 - Aberdeen. IEEE, jun 2017, pp. 1-7.

[8] M. Ludvigsen, S. M. Albrektsen, K. Cisek, T. A. Johansen, P. Norgren, R. Skjetne, A. Zolich, P. S. Dias, A. S. Ferreira, J. B. de Sousa, T. O. Fossum, O. Sture, T. Robekk Krogstad, O. Midtgaard, V. Hovstein, and E. Vagsholm, "Network of heterogeneous autonomous vehicles for marine research and management," in OCEANS 2016 MTS/IEEE Monterey. IEEE, sep 2016, pp. 1-7.

[9] J. B. de Sousa and F. L. Pereira, "Coordination Challenges in Networked Vehicle Systems: Are We Missing Something?" in Coordination Control of Distributed Systems, J. H. van Schuppen and T. Villa, Eds. Springer International Publishing, 2015, pp. 27-34

[10] J. B. de Sousa, P. McGuillivary, J. Vicente, M. N. Bento, J. A. P. Morgado, M. M. Matos, R. A. G. Bencatel, and P. M. de Oliveira, Unmanned Aircraft Systems for Maritime Operations. Dordrecht: Springer Netherlands, 2015, pp. 2787-2811.

[11] F. Py, J. Pinto, M. Silva, T. A. Johansen, J. B. de Sousa, and K. Rajan, "EUROPtus: A Mixed-initiative Controller for Multi-Vehicle Oceanographic Field Experiments," International Symposium on Experimental Robotics, no. October, pp. 1-10, 2016.

[12] J. Pinto, P. Sousa Dias, J. Pereira, R. Caldas, T. Rodrigues, J. B. de Sousa, F. Py, and K. Rajan, "Mixed-Initiative Interaction for Tracking of Ocean Sunfish," IFAC-PapersOnLine, vol. 48, no. 2, pp. 94-99, 2015.

[13] L. Chrpa, J. Pinto, M. A. Ribeiro, F. Py, J. Sousa, and K. Rajan, "On mixed-initiative planning and control for Autonomous underwater vehicles," in 2015 IEEE/RSJ International Conference on Intelligent Robots and Systems (IROS). IEEE, sep 2015, pp. 1685-1690.

[14] R. Gonçalves, A. S. Ferreira, J. Pinto, J. B. Sousa, and G. Gonçalves, "Authority sharing in mixed initiative control of multiple uninhabited aerial vehicles," Engineering Psychology and Cognitive Ergonomics, pp. 530-539, 2011.

[15] C. Fuchs, A. S. Ferreira, J. B. de Sousa, and G. Gonçalves, "Adaptive Consoles for Supervisory Control of Multiple Unmanned Aerial Vehicles," in Human-Computer Interaction. Interaction Modalities and Techniques, ser. Lecture Notes in Computer Science, M. Kurosu, Ed. Springer Berlin Heidelberg, 2013, vol. 8007, pp. 678-687.

[16] D. Kidston and T. Kunz, "Challenges and opportunities in managing maritime networks," IEEE Communications Magazine, vol. 46, no. 10, pp. 162-168, oct 2008. [Online].
Available: http://portal.acm.org/citation.cfm?doid=1071246.1071304 http://ieeexplore.ieee.org/document/4644135/

[17] L. Gupta, R. Jain, and G. Vaszkun, "Survey of Important Issues in UAV Communication Networks," IEEE Communications Surveys \& Tutorials, vol. 18, no. 2, pp. 1123-1152, 2016. [Online]. Available: http://ieeexplore.ieee.org/document/7317490/ http://dx.doi.org/10.1109/COMST.2015.2495297

[18] A. S. Ferreira, J. Pereira, P. Sousa Dias, J. B. de Sousa, T. A Johansen, P. Lourenco, and V. E. Hovstein, "Managing communication challenges in vehicle networks for remote maritime operations," in OCEANS 2017 - Aberdeen. IEEE, jun 2017, pp. 1-7. [Online]. Available: http://ieeexplore.ieee.org/document/8084770/

[19] D. Palma, A. Zolich, Y. Jiang, and T. A. Johansen, "Unmanned Aerial Vehicles as Data Mules: An Experimental Assessment," IEEE Access, vol. 5, no. Figure 1, pp. 24716-24726, 2017. [Online] Available: http://ieeexplore.ieee.org/document/8094228/

[20] J. B. de Sousa, K. Rajan, J. Pereira, M. Incze, J. Pinto, J. Alves, P. Lourenço, K. Pelekanakis, M. Galocha, J. Fontes, A. Munafò, T. A. Johansen, and R. Petroccia, "Rapid Environmental Picture Atlantic exercise 2015 : a field report," in MTS/IEEE OCEANS Conference, 2015.

[21] A. S. Ferreira, J. Pinto, P. Sousa Dias, and J. B. de Sousa, "The LSTS software toolchain for persistent maritime operations applied through vehicular ad-hoc networks," in 2017 International Conference on Unmanned Aircraft Systems (ICUAS). IEEE, jun 2017, pp. 609616.

[22] R. Martins, J. B. de Sousa, C. C. Afonso, and M. Incze, "REP10 AUV: Shallow water operations with heterogeneous autonomous vehicles," in OCEANS 2011 IEEE - Spain. IEEE, jun 2011, pp. 1-6. [Online]. Available: http://ieeexplore.ieee.org/document/6003568/

[23] T. Marques, K. Lima, M. Ribeiro, A. S. Ferreira, J. B. Sousa, and R. Mendes, "Characterization of Highly Dynamic Coastal Environments, Employing Teams of Heterogeneous Vehicles: A Holistic Case Study," in 2018 OCEANS - MTS/IEEE Kobe TechnoOceans (OTO). IEEE, may 2018, pp. 1-8. [Online]. Available: https://ieeexplore.iee.org/document/8559107/

[24] M. Ribeiro, J. Galante, J. Teixeira, and J. B. de Sousa, "Use of a UAV as an acoustic communication relay system," in 2018 International Conference on Unmanned Aircraft Systems (ICUAS). IEEE, jun 2018, pp. 788-795. [Online]. Available: https://ieeexplore.ieee.org/document/8453407/

[25] OptiMOSTM 5 Power-Transistor, $80 \mathrm{~V}$, Infineon Technologies AG, 2 2015, rev. 2.1.

26] Arducopter https://github.com/ArduPilot/ardupilot/tree/master/ArduCopter.

[27] R. Martins, P. S. Dias, E. R. B. Marques, J. Pinto, J. B. Sousa, and F. L. Pereira, "IMC: A communication protocol for networked vehicles and sensors," in OCEANS 2009-EUROPE. IEEE, may 2009, pp. 1-6.

[28] P. S. Dias, G. Gonçalves, R. M. F. Gomes, J. B. Sousa, J. Pinto, J. Pereira, and F. L. Pereira, "Mission planning and specification in the Neptus framework," in Proceedings 2006 IEEE International Conference on Robotics and Automation, 2006. ICRA 2006. IEEE, 2006, pp. 3220-3225.

[29] P. S. Dias, S. Fraga, R. M. F. Gomes, G. Gonçalves, F. L. Pereira, J. Pinto, and J. B. Sousa, "Neptus - a framework to support multiple vehicle operation," in Europe Oceans 2005, vol. 2. IEEE, 2005, pp. 963-968. 